\title{
Pseudo almost periodic solutions for quaternion-valued cellular neural networks with discrete and distributed delays
}

\author{
Xiaofang Meng ${ }^{1}$ and Yongkun $\mathrm{Li}^{1 *}$ (D)
}

"Correspondence: yklie@ynu.edu.cn

'Department of Mathematics, Yunnan University, Kunming,

People's Republic of China

\section{Springer}

\begin{abstract}
This paper is concerned with a class of quaternion-valued cellular neural networks with discrete and distributed delays. By using the exponential dichotomy of linear systems and a fixed point theorem, sufficient conditions are derived for the existence and global exponential stability of pseudo almost periodic solutions of this class of neural networks. Finally, a numerical example is given to illustrate the feasibility of the obtained results.
\end{abstract}

MSC: 34K14; 34K20; 92B20

Keywords: Quaternion-valued cellular neural networks; Distributed delay; Pseudo almost periodic solutions; Exponent stability

\section{Introduction}

Since Chua and Yang proposed cellular neural networks (CNNs) in 1988 [1], various dynamical behaviors of $\mathrm{CNNs}$, such as the existence and stability of the equilibrium, periodic solutions, anti-periodic solutions, almost periodic solutions, and pseudo-almost periodic solutions, have been studied by many scholars [2-15].

On the one hand, quaternion-valued neural networks (QVNNs), as an extension of the complex-valued neural networks (CVNNs), can deal with multi-level information and require only half the connection weight parameters of CVNNs [16]. Moreover, compared with CVNNs, QVNNs perform more prominently when it comes to geometrical transformations, like 2D affine transformations or 3D affine transformations. 3D geometric affine transformations can be represented efficiently and compactly based on QVNNs, especially spatial rotation [17]. Since the multiplication of quaternion is not commutative due to Hamilton rules: $i j=-j i=k, j k=-k j=i, k i=-i k=j, i^{2}=j^{2}=k^{2}=i j k=-1$, the analysis for QVCNNs becomes difficult. However, with the continuous development of the theory of quaternion, there are some results about the dynamics of QVNNs. For example, the authors of $[18,19]$ studied the existence and global exponential stability of equilibrium point for QVNNs; the authors of [20] investigated the robust stability of QVNNs with time delays and parameter uncertainties; the authors of [21] considered the existence and stability of pseudo almost periodic solutions for a class of QVCNNs on time scales by a special decomposition method; the authors of $[22,23]$ investigated the existence and global $\mu$ stability of an equilibrium point for QVNNs; the authors of [24] dealt with the existence

(c) The Author(s) 2018. This article is distributed under the terms of the Creative Commons Attribution 4.0 International License (http://creativecommons.org/licenses/by/4.0/), which permits unrestricted use, distribution, and reproduction in any medium, provided you give appropriate credit to the original author(s) and the source, provide a link to the Creative Commons license, and indicate if changes were made. 
and stability of periodic solutions for QVCNNs by using a continuation theorem of coincidence degree theory; the authors of [25] studied the almost periodic synchronization for QVCNNs. Although non-autonomous neural networks are more general and practical than the autonomous ones, up to now, there have been only few results about the dynamic behaviors of non-autonomous QVNNs.

On the other hand, it is well known that the periodicity, almost periodicity, pseudo almost periodicity, and so on are the very important dynamics for non-autonomous systems $[10,12,26]$. Moreover, the almost periodicity is more general than the periodicity. In addition, the pseudo almost periodicity is a natural generalization of almost periodicity. In the past few years, the pseudo almost periodicity of real-valued neural networks (RVNNs) has been studied by many authors [13-15, 27-34]. Besides, as we all know, time delay is universal and can change the dynamical behavior of the system under consideration [3, $5,29,30,35,36]$. Therefore, it is important and necessary to consider the neural network model with time delay. However, to the best of our knowledge, there is no paper published on the existence and stability of pseudo almost periodic solutions for quaternion-valued cellular neural networks (QVCNNs) with discrete and distributed delays.

Motivated by the above, in this paper, we are concerned with the following QVCNN with discrete and distributed delays:

$$
\begin{aligned}
x_{p}^{\prime}(t)= & -c_{p}(t) x_{p}(t)+\sum_{q=1}^{n} a_{p q}(t) f_{q}\left(x_{q}\left(t-\tau_{p q}(t)\right)\right) \\
& +\sum_{q=1}^{n} b_{p q}(t) \int_{0}^{\infty} K_{p q}(u) g_{q}\left(x_{q}(t-u)\right) \mathrm{d} u+u_{p}(t),
\end{aligned}
$$

where $p \in\{1,2, \ldots, n\}:=\Lambda, x_{p}(t) \in \mathbb{Q}$ is the state vector of the $p$ th unit at time $t, c_{p}(t)>$ 0 represents the rate at which the $p$ th unit will reset its potential to the resting state in isolation when disconnected from the network and external inputs, $a_{p q}(t), b_{p q}(t) \in \mathbb{Q}$ are the synaptic weights of delayed feedback between the $p$ th neuron and the $q$ th neuron, $f_{q}, g_{q}: \mathbb{Q} \rightarrow \mathbb{Q}$ are the activation functions of signal transmission, $\tau_{p q}(t) \geq 0$ denotes the transmission delay, $u_{p}(t) \in \mathbb{Q}$ denotes the external input on the $p$ th neuron at time $t$.

Throughout this paper, we denote by $B C\left(\mathbb{R}, \mathbb{R}^{n}\right)$, the set of all bounded continuous functions from $\mathbb{R}$ to $\mathbb{R}^{n}$.

The initial value is given by

$$
x_{p}(s)=\phi_{p}(s), \quad s \in(-\infty, 0], p \in \Lambda,
$$

where $\phi_{p} \in B C((-\infty, 0], \mathbb{Q})$.

Our main aim in this paper is to study the existence and global exponential stability of pseudo almost periodic solutions of (1). The main contributions of this paper are listed as follows.

(1) To the best of our knowledge, this is the first time to study the existence and stability of pseudo almost periodic solutions for QVCNNs with discrete and distributed delays.

(2) The stability of QVNNs with distributed delays has not been reported yet. Therefore, our result about the stability of QVNNs is new, and most of the existing 
results about the stability of QVNNs are obtained by using the theory of linear matrix inequalities but ours are not.

(3) The method that we use to transform QVNNs into RVNNs is different from that used in [18, 20-23].

(4) QVCNN (1) contains RVCNNs and CVCNNs as its special cases.

Throughout this paper, $\mathbb{R}^{n \times n}, \mathbb{Q}^{n \times n}$ denote the set of all $n \times n$ real-valued and quaternion-valued matrices, respectively. The skew field of quaternion is denoted by

$$
\mathbb{Q}:=\left\{x=x^{R}+i x^{I}+j x^{J}+k x^{K}\right\}
$$

where $x^{R}, x^{I}, x^{J}, x^{K}$ are real numbers and the elements $i, j$, and $k$ obey Hamilton's multiplication rules.

For the convenience, we will introduce the notations: $\bar{h}=\sup _{t \in \mathbb{R}}|h(t)|, \underline{h}=\inf _{t \in \mathbb{R}}|h(t)|$, where $h(t)$ is a bounded continuous function.

This paper is organized as follows. In Sect. 2, we introduce some definitions, make some preparations for later sections. In Sect. 3, by utilizing Banach's fixed point theorem and differential inequality techniques, we establish the existence and global exponential stability of pseudo almost periodic solutions of (1). In Sect. 4, we give an example to demonstrate the feasibility of our results. This paper ends with a brief conclusion in Sect. 5 .

\section{Preliminaries}

In this section, we shall first recall some fundamental definitions, lemmas which are used in what follows.

Definition 1 ([37]) A function $u \in B C\left(\mathbb{R}, \mathbb{R}^{n}\right)$ is said to be almost periodic if, for any $\epsilon>0$, it is possible to find a real number $l=l(\epsilon)>0$, for any interval with length $l(\epsilon)$, there exists a number $\tau=\tau(\epsilon)$ in this interval such that $|u(t+\tau)-u(t)|<\epsilon$ for all $t \in \mathbb{R}$. The collection of such functions will be denoted by $A P\left(\mathbb{R}, \mathbb{R}^{n}\right)$.

Let

$$
\operatorname{PAP}_{0}\left(\mathbb{R}, \mathbb{R}^{n}\right)=\left\{f \in B C\left(\mathbb{R}, \mathbb{R}^{n}\right) \mid \lim _{r \rightarrow+\infty} \frac{1}{2 r} \int_{-r}^{r}\|f(t)\| \mathrm{d} t=0\right\}
$$

Definition 2 ([38,39]) A function $f \in B C\left(\mathbb{R}, \mathbb{R}^{n}\right)$ is called pseudo almost periodic if it can be expressed as $f=f_{1}+f_{0}$, where $f_{1} \in A P\left(\mathbb{R}, \mathbb{R}^{n}\right)$ and $f_{0} \in P A P_{0}\left(\mathbb{R}, \mathbb{R}^{n}\right)$. The collection of such functions will be denoted by $P A P\left(\mathbb{R}, \mathbb{R}^{n}\right)$.

From the above definitions, it is easy to see that $A P\left(\mathbb{R}, \mathbb{R}^{n}\right) \subset P A P\left(\mathbb{R}, \mathbb{R}^{n}\right)$.

Definition 3 A quaternion-valued function $x=x^{R}+i x^{I}+j x^{J}+k x^{K} \in B C\left(\mathbb{R}, \mathbb{Q}^{n}\right)$ is called a pseudo almost periodic function if, for every $l \in\{R, I, J, K\}:=E, x^{l} \in P A P\left(\mathbb{R}, \mathbb{R}^{n}\right)$.

Definition $4([38,39])$ The system

$$
x^{\prime}(t)=A(t) x(t)
$$


is said to admit an exponential dichotomy if there exist a projection $P$ and positive constants $\alpha, \beta$ such that the fundamental solution matrix $X(t)$ satisfies

$$
\begin{aligned}
& \left|X(t) P X^{-1}(s)\right| \leq \beta e^{-\alpha(t-s)}, \quad t \geq s, \\
& \left|X(t)(I-P) X^{-1}(s)\right| \leq \beta e^{-\alpha(s-t)}, \quad t \leq s .
\end{aligned}
$$

Consider the following pseudo almost periodic system:

$$
x^{\prime}(t)=A(t) x(t)+f(t)
$$

where $A(t)$ is an almost periodic matrix function, $f(t)$ is a pseudo almost periodic vector function.

Lemma $1([38,39])$ If the linear system (2) admits an exponential dichotomy, then system (3) has a unique pseudo almost periodic solution:

$$
x(t)=\int_{-\infty}^{t} X(t) P X^{-1}(s) f(s) \mathrm{d} s-\int_{t}^{+\infty} X(t)(I-P) X^{-1}(s) f(s) \mathrm{d} s,
$$

where $X(t)$ is the fundamental solution matrix of (2).

Lemma $2([38,39])$ Let $c_{p}(t)$ be an almost periodic function on $\mathbb{R}$ and

$$
M\left[c_{p}\right]=\lim _{T \rightarrow \infty} \frac{1}{T} \int_{t}^{t+T} c_{p}(s) \mathrm{d} s>0, \quad p \in \Lambda .
$$

Then the linear system

$$
x^{\prime}(t)=\operatorname{diag}\left(-c_{1}(t),-c_{2}(t), \ldots,-c_{n}(t)\right) x(t)
$$

admits an exponential dichotomy on $\mathbb{R}$.

In order to decompose the quaternion-valued system (1) into a real-valued system, we need the following assumption:

$\left(S_{1}\right)$ Let $x_{p}=x_{p}^{R}+i x_{p}^{I}+j x_{p}^{J}+k x_{p}^{K}, x_{p}^{l} \in \mathbb{R}, l \in E$. Then the activation functions $f_{q}\left(x_{q}\right)$ and $g_{q}\left(x_{q}\right)$ of (1) can be expressed as

$$
\begin{aligned}
f_{q}\left(x_{q}\right)= & f_{q}^{R}\left(x_{q}^{R}, x_{q}^{I}, x_{q}^{J}, x_{q}^{K}\right) \\
& +i f_{q}^{I}\left(x_{q}^{R}, x_{q}^{I}, x_{q}^{J}, x_{q}^{K}\right)+j f_{q}^{J}\left(x_{q}^{R}, x_{q}^{I}, x_{q}^{J}, x_{q}^{K}\right)+k f_{q}^{K}\left(x_{q}^{R}, x_{q}^{I}, x_{q}^{J}, x_{q}^{K}\right), \\
g_{q}\left(x_{q}\right)= & g_{q}^{R}\left(x_{q}^{R}, x_{q}^{I}, x_{q}^{J}, x_{q}^{K}\right)+i g_{q}^{I}\left(x_{q}^{R}, x_{q}^{I}, x_{q}^{J}, x_{q}^{K}\right)+j g_{q}^{J}\left(x_{q}^{R}, x_{q}^{I}, x_{q}^{J}, x_{q}^{K}\right) \\
& +k g_{q}^{K}\left(x_{q}^{R}, x_{q}^{I}, x_{q}^{J}, x_{q}^{K}\right),
\end{aligned}
$$

where $f_{q}^{l}, g_{q}^{l}: \mathbb{R}^{4} \rightarrow \mathbb{R}, p \in \Lambda, l \in E$. 
Under assumption $\left(S_{1}\right)$, system (1) can be decomposed into the following four realvalued sub-systems:

$$
\begin{aligned}
& \left(x_{p}^{R}(t)\right)^{\prime}=-c_{p}(t) x_{p}^{R}(t)+\sum_{q=1}^{n}\left(a_{p q}^{R}(t) f_{q}^{R}[t, x]-a_{p q}^{I}(t) f_{q}^{I}[t, x]\right. \\
& \left.-a_{p q}^{J}(t) f_{q}^{J}[t, x]-a_{p q}^{K}(t) f_{q}^{K}[t, x]\right)+\sum_{q=1}^{n}\left(b_{p q}^{R}(t) \int_{0}^{\infty} K_{p q}(u)\right. \\
& \times g_{q}^{R}[t, u, x] \mathrm{d} u-b_{p q}^{I}(t) \int_{0}^{\infty} K_{p q}(u) g_{q}^{I}[t, u, x] \mathrm{d} u \\
& -b_{p q}^{J}(t) \int_{0}^{\infty} K_{p q}(u) g_{q}^{J}[t, u, x] \mathrm{d} u-b_{p q}^{K}(t) \int_{0}^{\infty} K_{p q}(u) \\
& \left.\times g_{q}^{K}[t, u, x] \mathrm{d} u\right)+u_{p}^{R}(t), \\
& \left(x_{p}^{I}(t)\right)^{\prime}=-c_{p}(t) x_{p}^{I}(t)+\sum_{q=1}^{n}\left(a_{p q}^{R}(t) f_{q}^{I}[t, x]+a_{p q}^{I}(t) f_{q}^{R}[t, x]\right. \\
& \left.+a_{p q}^{J}(t) f_{q}^{K}[t, x]-a_{p q}^{K}(t) f_{q}^{J}[t, x]\right)+\sum_{q=1}^{n}\left(b_{p q}^{R}(t) \int_{0}^{\infty} K_{p q}(u)\right. \\
& \times g_{q}^{I}[t, u, x] \mathrm{d} u+b_{p q}^{I}(t) \int_{0}^{\infty} K_{p q}(u) g_{q}^{R}[t, u, x] \mathrm{d} u \\
& +b_{p q}^{J}(t) \int_{0}^{\infty} K_{p q}(u) g_{q}^{K}[t, u, x] \mathrm{d} u-b_{p q}^{K}(t) \int_{0}^{\infty} K_{p q}(u) \\
& \left.\times g_{q}^{J}[t, u, x] \mathrm{d} u\right)+u_{p}^{I}(t), \\
& \left(x_{p}^{J}(t)\right)^{\prime}=-c_{p}(t) x_{p}^{J}(t)+\sum_{q=1}^{n}\left(a_{p q}^{R}(t) f_{q}^{J}[t, x]+a_{p q}^{J}(t) f_{q}^{R}[t, x]\right. \\
& \left.-a_{p q}^{I}(t) f_{q}^{K}[t, x]+a_{p q}^{K}(t) f_{q}^{I}[t, x]\right)+\sum_{q=1}^{n}\left(b_{p q}^{R}(t) \int_{0}^{\infty} K_{p q}(u)\right. \\
& \times g_{q}^{J}[t, u, x] \mathrm{d} u+b_{p q}^{J}(t) \int_{0}^{\infty} K_{p q}(u) g_{q}^{R}[t, u, x] \mathrm{d} u \\
& -b_{p q}^{I}(t) \int_{0}^{\infty} K_{p q}(u) g_{q}^{K}[t, u, x] \mathrm{d} u+b_{p q}^{K}(t) \int_{0}^{\infty} K_{p q}(u) \\
& \left.\times g_{q}^{I}[t, u, x] \mathrm{d} u\right)+u_{p}^{J}(t), \\
& \left(x_{p}^{K}(t)\right)^{\prime}=-c_{p}(t) x_{p}^{K}(t)+\sum_{q=1}^{n}\left(a_{p q}^{R}(t) f_{q}^{K}[t, x]+a_{p q}^{K}(t) f_{q}^{R}[t, x]\right. \\
& \left.+a_{p q}^{I}(t) f_{q}^{J}[t, x]-a_{p q}^{J}(t) f_{q}^{I}[t, x]\right)+\sum_{q=1}^{n}\left(b_{p q}^{R}(t) \int_{0}^{\infty} K_{p q}(u)\right. \\
& \times g_{q}^{K}[t, u, x] \mathrm{d} u+b_{p q}^{K}(t) \int_{0}^{\infty} K_{p q}(u) g_{q}^{R}[t, u, x] \mathrm{d} u \\
& +b_{p q}^{I}(t) \int_{0}^{\infty} K_{p q}(u) g_{q}^{J}[t, u, x] \mathrm{d} u-b_{p q}^{J}(t) \int_{0}^{\infty} K_{p q}(u)
\end{aligned}
$$




$$
\left.\times g_{q}^{I}[t, u, x] \mathrm{d} u\right)+u_{p}^{K}(t)
$$

where $f_{q}^{l}[t, x] \triangleq f_{q}^{l}\left(x_{q}^{R}\left(t-\tau_{p q}(t)\right), x_{q}^{I}\left(t-\tau_{p q}(t)\right), x_{q}^{J}\left(t-\tau_{p q}(t)\right), x_{q}^{K}\left(t-\tau_{p q}(t)\right)\right), g_{q}^{l}[t, u, x] \triangleq$ $g_{q}^{l}\left(x_{q}^{R}(t-u),\left(x_{q}^{I}(t-u)\right),\left(x_{q}^{J}(t-u)\right),\left(x_{q}^{K}(t-u)\right)\right)$, and

$$
\begin{aligned}
& a_{p q}(t)=a_{p q}^{R}(t)+i a_{p q}^{I}(t)+j a_{p q}^{J}(t)+k a_{p q}^{K}(t), \\
& b_{p q}(t)=b_{p q}^{R}(t)+i b_{p q}^{I}(t)+j b_{p q}^{J}(t)+k b_{p q}^{K}(t), \\
& u_{p}(t)=u_{p}^{R}(t)+i u_{p}^{I}(t)+j u_{p}^{I}(t)+k u_{p}^{K}(t) .
\end{aligned}
$$

According to (4)-(7), one can obtain that

$$
\begin{aligned}
X_{p}^{\prime}(t)= & -c_{p}(t) X_{p}(t)+\sum_{q=1}^{n} A_{p q}(t) F_{q}[t, x] \\
& +\sum_{q=1}^{n} B_{p q}(t) \int_{0}^{\infty} K_{p q}(u) G_{q}[t, u, x] \mathrm{d} u+U_{p}(t), \quad p \in \Lambda,
\end{aligned}
$$

where

$$
\begin{aligned}
& A_{p q}(t)=\left(\begin{array}{cccc}
a_{p q}^{R}(t) & -a_{p q}^{I}(t) & -a_{p q}^{J}(t) & -a_{p q}^{K}(t) \\
a_{p q}^{I}(t) & a_{p q}^{R}(t) & -a_{p q}^{K}(t) & a_{p q}^{I}(t) \\
a_{p q}^{I}(t) & a_{p q}^{K}(t) & a_{p q}^{R}(t) & -a_{p q}^{I}(t) \\
a_{p q}^{K}(t) & -a_{p q}^{I}(t) & a_{p q}^{I}(t) & a_{p q}^{R}(t)
\end{array}\right) \\
& B_{p q}(t)=\left(\begin{array}{cccc}
b_{p q}^{R}(t) & -b_{p q}^{I}(t) & -b_{p q}^{J}(t) & -b_{p q}^{K}(t) \\
b_{p q}^{I}(t) & b_{p q}^{R}(t) & -b_{p q}^{K}(t) & b_{p q}^{J}(t) \\
b_{p q}^{I}(t) & b_{p q}^{K}(t) & b_{p q}^{R}(t) & -b_{p q}^{I}(t) \\
b_{p q}^{K}(t) & -b_{p q}^{J}(t) & b_{p q}^{I}(t) & b_{p q}^{R}(t)
\end{array}\right), \quad X_{p}(t)=\left(\begin{array}{c}
x_{p}^{R}(t) \\
x_{p}^{I}(t) \\
x_{p}^{J}(t) \\
x_{p}^{K}(t)
\end{array}\right), \\
& U_{p}(t)=\left(\begin{array}{c}
u_{p}^{R}(t) \\
u_{p}^{I}(t) \\
u_{p}^{J}(t) \\
u_{p}^{K}(t)
\end{array}\right), \quad F_{q}[t, x]=\left(\begin{array}{c}
f_{q}^{R}[t, x] \\
f_{q}^{I}[t, x] \\
f_{q}^{J}[t, x] \\
f_{q}^{K}[t, x]
\end{array}\right), \quad G_{q}[t, u, x]=\left(\begin{array}{c}
g_{q}^{R}[t, u, x] \\
g_{q}^{I}[t, u, x] \\
g_{q}^{J}[t, u, x] \\
g_{q}^{K}[t, u, x]
\end{array}\right) .
\end{aligned}
$$

The initial condition associated with (8) is of the form

$$
X_{p}(s)=\Phi_{p}(s), \quad p \in \Lambda, s \in(-\infty, 0]
$$

where

$$
\Phi_{p}(s)=\left(\phi_{p}^{R}(s), \phi_{p}^{I}(s), \phi_{p}^{J}(s), \phi_{p}^{K}(s)\right)^{T}
$$

and $\phi_{p}^{l}(s) \in B C((-\infty, 0], \mathbb{R}), p \in \Lambda, l \in E$.

Remark 1 Under assumption $\left(S_{1}\right)$, it is easy to see that if $X=\left(x_{1}^{R}, x_{1}^{I}, x_{1}^{J}, x_{1}^{K}, \ldots, x_{n}^{R}, x_{n}^{I}, x_{n}^{J}\right.$, $\left.x_{n}^{K}\right)^{T} \in \mathbb{R}^{4 n}$ is a solution of system (8), then $x=\left(X_{1}, X_{2}, \ldots, X_{n}\right)^{T}$ is a solution of system (1), and vice visa, where $X_{p}=x_{p}^{R}+i x_{p}^{I}+j x_{p}^{J}+k x_{p}^{K}, p \in \Lambda$. Therefore, to find a solution for system 
(1) is equivalent to finding one for system (8). To study the stability of solutions of system (1), we only need to investigate the stability of solutions of system (8).

\section{Main results}

In this section, we establish the existence and global exponential stability of pseudo almost periodic solutions of system (8).

Let $\mathbb{X}=\left\{f(t) \mid f \in \operatorname{PAP}\left(\mathbb{R}, \mathbb{R}^{4 n}\right)\right\}$ with the norm $\|f\|_{\mathbb{X}}=\sup _{t \in \mathbb{R}}\|f(t)\|$, where $\|f(t)\|=$ $\max _{1 \leq h \leq 4 n}\left\{\left|f_{h}(t)\right|\right\}$, then $\mathbb{X}$ is a Banach space.

In the following, we assume that the following conditions hold:

$\left(S_{2}\right)$ There exist positive constants $\alpha_{q}^{l}, \beta_{q}^{l}$ such that

$$
\begin{aligned}
& \left|f_{q}^{l}\left(x_{q}^{R}, x_{q}^{I}, x_{q}^{J}, x_{q}^{K}\right)-f_{q}^{l}\left(y_{q}^{R}, y_{q}^{I}, y_{q}^{J}, y_{q}^{K}\right)\right| \\
& \quad \leq \alpha_{q}^{R}\left|x_{q}^{R}-y_{q}^{R}\right|+\alpha_{q}^{I}\left|x_{q}^{I}-y_{q}^{I}\right|+\alpha_{q}^{J}\left|x_{q}^{J}-y_{q}^{J}\right|+\alpha_{q}^{K}\left|x_{q}^{K}-y_{q}^{K}\right|, \\
& \left|g_{q}^{l}\left(x_{q}^{R}, x_{q}^{I}, x_{q}^{J}, x_{q}^{K}\right)-g_{q}^{l}\left(y_{q}^{R}, y_{q}^{I}, y_{q}^{J}, y_{q}^{K}\right)\right| \\
& \quad \leq \beta_{q}^{R}\left|x_{q}^{R}-y_{q}^{R}\right|+\beta_{q}^{I}\left|x_{q}^{I}-y_{q}^{I}\right|+\beta_{q}^{J}\left|x_{q}^{J}-y_{q}^{J}\right|+\beta_{q}^{K}\left|x_{q}^{K}-y_{q}^{K}\right|
\end{aligned}
$$

and $f_{q}^{l}(0,0,0,0)=g_{q}^{l}(0,0,0,0)=0$, where $p \in \Lambda, l \in E$.

$\left(S_{3}\right)$ The function $c_{p} \in C\left(\mathbb{R}, \mathbb{R}^{+}\right)$with $M\left[c_{p}\right]>0$ is almost periodic, $U_{p} \in C\left(\mathbb{R}, \mathbb{R}^{4 \times 1}\right)$, $A_{p q}, B_{p q} \in C\left(\mathbb{R}, \mathbb{R}^{4 \times 4}\right)$, and $\tau_{p q} \in C\left(\mathbb{R}, \mathbb{R}^{+}\right)$are pseudo almost periodic, where $p, q \in$ $\Lambda$.

$\left(S_{4}\right)$ The delay kernel $K_{p q}:[0, \infty) \rightarrow \mathbb{R}$ is continuous and integrable with $0 \leq$ $\int_{0}^{\infty}\left|K_{p q}(u)\right| \mathrm{d} u \leq \bar{K}_{p q}$, where $p, q \in \Lambda$.

$\left(S_{5}\right)$ There exists a constant $\kappa$ such that

$$
\max _{p \in \Lambda}\left\{\max _{l \in E}\left\{\frac{\Theta_{p} \kappa+\bar{u}_{p}^{l}}{\underline{c}_{p}}\right\}\right\} \leq \kappa, \quad \max _{p \in \Lambda}\left\{\frac{\Theta_{p}}{\underline{c}_{p}}\right\}:=\rho<1,
$$

where

$$
\begin{aligned}
& \Theta_{p}=V_{p}+W_{p}, \quad p \in \Lambda, \\
& V_{p}=\sum_{q=1}^{n}\left(\bar{a}_{p q}^{R}+\bar{a}_{p q}^{I}+\bar{a}_{p q}^{I}+\bar{a}_{p q}^{K}\right)\left(\alpha_{q}^{R}+\alpha_{q}^{I}+\alpha_{q}^{J}+\alpha_{q}^{K}\right), \quad p \in \Lambda, \\
& W_{p}=\sum_{q=1}^{n} \bar{K}_{p q}\left(\bar{b}_{p q}^{R}+\bar{b}_{p q}^{I}+\bar{b}_{p q}^{I}+\bar{b}_{p q}^{K}\right)\left(\beta_{q}^{R}+\beta_{q}^{I}+\beta_{q}^{I}+\beta_{q}^{K}\right), \quad p \in \Lambda .
\end{aligned}
$$

Theorem 1 Suppose that $\left(S_{1}\right)-\left(S_{5}\right)$ hold. Then system (8) has a unique pseudo almost periodic solution in the region $\mathbb{X}^{*}=\left\{\varphi \mid \varphi \in \mathbb{X},\|\varphi\|_{\mathbb{X}} \leq \kappa\right\}$.

Proof Let $\varphi=\left(\varphi_{1}^{R}, \varphi_{1}^{I}, \varphi_{1}^{J}, \varphi_{1}^{K}, \ldots, \varphi_{n}^{R}, \varphi_{n}^{I}, \varphi_{n}^{J}, \varphi_{n}^{K}\right)^{T} \in \mathbb{X}$. Obviously, $\left(S_{1}\right)$ implies that $F_{q}[t, \varphi]$ and $G_{q}[t, u, \varphi]$ are uniformly continuous functions on $\mathbb{R}$ for $q \in \Lambda$. Set $h(t, z)=\varphi_{q}(t-z)$ $(q \in \Lambda)$, where $\varphi_{q}(t-z)=\left(\varphi_{q}^{R}(t-z), \varphi_{q}^{I}(t-z), \varphi_{q}^{J}(t-z), \varphi_{q}^{K}(t-z)\right)$. By Theorem 5.3 in [40] and Definition 5.7 in [40], we can obtain that $h \in P A P(\mathbb{R} \times \Omega)$ and $h$ is continuous in $z \in K$ and uniformly in $t \in \mathbb{R}$ for all compact subset $K$ of $\Omega \subset \mathbb{R}$. This, together with $\tau_{p q} \in P A P\left(\mathbb{R}, \mathbb{R}^{+}\right)$ 
and Theorem 5.11 in [40], implies that

$$
\varphi_{q}\left(t-\tau_{p q}(t)\right) \in P A P\left(\mathbb{R}, \mathbb{R}^{4}\right), \quad p, q \in \Lambda .
$$

Again from Corollary 5.4 in [40], we have

$$
F_{q}[t, \varphi] \in P A P\left(\mathbb{R}^{4}, \mathbb{R}^{4 \times 1}\right), \quad q \in \Lambda,
$$

which implies that

$$
\sum_{q=1}^{n} A_{p q}(t) F_{q}[t, \varphi] \in \operatorname{PAP}\left(\mathbb{R}, \mathbb{R}^{4 \times 1}\right), \quad p, q \in \Lambda
$$

By a similar argument as that in the proof of Lemma 2.3 in [13], one can obtain that

$$
\sum_{q=1}^{n} B_{p q}(t) \int_{0}^{\infty} K_{p q}(u) G_{q}\left(\varphi_{q}(t-u)\right) \mathrm{d} u \in \operatorname{PAP}\left(\mathbb{R}, \mathbb{R}^{4 \times 1}\right), \quad p \in \Lambda .
$$

For any $\varphi \in \mathbb{X}$, consider the following linear system:

$$
\begin{aligned}
X_{p}^{\prime}(t)= & -c_{p}(t) X_{p}(t)+\sum_{q=1}^{n} A_{p q}(t) F_{q}[t, \varphi] \\
& +\sum_{q=1}^{n} B_{p q}(t) \int_{0}^{\infty} K_{p q}(u) G_{q}[t, u, \varphi] \mathrm{d} u+U_{p}(t), \quad p \in \Lambda .
\end{aligned}
$$

In view of Lemma 2, we can conclude that the linear system

$$
X_{p}^{\prime}(t)=-c_{p}(t) X_{p}(t), \quad p \in \Lambda
$$

admits an exponential dichotomy. Furthermore, by Lemma 1, we obtain that system (9) has exactly one pseudo periodic almost solution:

$$
X^{\varphi}=\left(X_{1}^{\varphi}, X_{2}^{\varphi}, \ldots, X_{n}^{\varphi}\right),
$$

where

$$
\begin{aligned}
X_{p}^{\varphi}(t)= & \int_{-\infty}^{t} e^{-\int_{s}^{t} c_{p}(u) \mathrm{d} u}\left(\sum_{q=1}^{n} A_{p q}(s) F_{q}[s, \varphi]\right. \\
& \left.+\sum_{q=1}^{n} B_{p q}(s) \int_{0}^{\infty} K_{p q}(u) G_{q}[s, u, \varphi] \mathrm{d} u+U_{p}(s)\right) \mathrm{d} s, \quad p \in \Lambda .
\end{aligned}
$$

Define a mapping $T: \mathbb{X} \rightarrow \mathbb{X}$ by setting $(T \varphi)(t)=X^{\varphi}(t), \forall \varphi \in \mathbb{X}$. Obviously, $\mathbb{X}^{*}$ is a closed convex subset of $\mathbb{X}$. 
Now, we prove that the mapping $T$ is a self-mapping from $\mathbb{X}^{*}$ to $\mathbb{X}^{*}$. In fact, for $\forall \varphi \in \mathbb{X}^{*}$, we have

$$
\begin{aligned}
& \sup _{t \in \mathbb{R}}\left|(T \varphi)_{p}^{R}(t)\right| \\
& =\sup _{t \in \mathbb{R}} \mid \int_{-\infty}^{t} e^{-\int_{s}^{t} c_{p}(u) \mathrm{d} u}\left(\sum _ { q = 1 } ^ { n } \left(a_{p q}^{R}(s) f_{q}^{R}[s, \phi]-a_{p q}^{I}(s) f_{q}^{I}[s, \phi]\right.\right. \\
& \left.-a_{p q}^{J}(s) f_{q}^{J}[s, \phi]-a_{p q}^{K}(s) f_{q}^{K}[s, \phi]\right)+\sum_{q=1}^{n}\left(b_{p q}^{R}(s) \int_{0}^{\infty} K_{p q}(u) \tilde{g}_{q}^{R} \mathrm{~d} u\right. \\
& -b_{p q}^{I}(s) \int_{0}^{\infty} K_{p q}(u) \tilde{g}_{q}^{I} \mathrm{~d} u-b_{p q}^{J}(s) \int_{0}^{\infty} K_{p q}(u) \tilde{g}_{q}^{J} \mathrm{~d} u \\
& \left.\left.-b_{p q}^{K}(s) \int_{0}^{\infty} K_{p q}(u) \tilde{g}_{q}^{K} \mathrm{~d} u\right)+u_{p}^{R}(s)\right) \mathrm{d} s \mid \\
& \leq \sup _{t \in \mathbb{R}} \int_{-\infty}^{t} e^{-\int_{s}^{t} c_{p}(u) \mathrm{d} u}\left(\sum_{q=1}^{n}\left(\bar{a}_{p q}^{R}+\bar{a}_{p q}^{I}+\bar{a}_{p q}^{I}+\bar{a}_{p q}^{K}\right)\right. \\
& \times\left(\alpha_{q}^{R}\left|\varphi_{q}^{R}\left(s-\tau_{p q}(s)\right)\right|+\alpha_{q}^{I}\left|\varphi_{q}^{I}\left(s-\tau_{p q}(s)\right)\right|+\alpha_{q}^{J}\left|\varphi_{q}^{J}\left(s-\tau_{p q}(s)\right)\right|\right. \\
& \left.+\alpha_{q}^{K}\left|\varphi_{q}^{K}\left(s-\tau_{p q}(s)\right)\right|\right)+\sum_{q=1}^{n}\left(\bar{b}_{p q}^{R}+\bar{b}_{p q}^{I}+\bar{b}_{p q}^{I}+\bar{b}_{p q}^{K}\right) \\
& \times \int_{0}^{\infty}\left|K_{p q}(u)\right|\left(\beta_{q}^{R}\left|\varphi_{q}^{R}(s-u)\right|+\beta_{q}^{I}\left|\varphi_{q}^{I}(s-u)\right|+\beta_{q}^{J}\left|\varphi_{q}^{J}(s-u)\right|\right. \\
& \left.\left.+\beta_{q}^{K}\left|\varphi_{q}^{K}(s-u)\right|\right) \mathrm{d} u+\bar{u}_{p}^{R}\right) \mathrm{d} s \\
& \leq \sup _{t \in \mathbb{R}} \int_{-\infty}^{t} e^{-\int_{s}^{t} c_{p}(u) \mathrm{d} u}\left(\sum _ { q = 1 } ^ { n } ( \overline { a } _ { p q } ^ { R } + \overline { a } _ { p q } ^ { I } + \overline { a } _ { p q } ^ { I } + \overline { a } _ { p q } ^ { K } ) \left(\alpha_{q}^{R}+\alpha_{q}^{I}+\alpha_{q}^{I}\right.\right. \\
& \left.+\alpha_{q}^{K}\right)\|\varphi\|_{\mathbb{X}}+\sum_{q=1}^{n} \bar{K}_{p q}\left(\bar{b}_{p q}^{R}+\bar{b}_{p q}^{I}+\bar{b}_{p q}^{I}+\bar{b}_{p q}^{K}\right)\left(\beta_{q}^{R}+\beta_{q}^{I}+\beta_{q}^{I}\right. \\
& \left.\left.+\beta_{q}^{K}\right)\|\varphi\|_{\mathbb{X}}+\bar{u}_{p}^{R}\right) \mathrm{d} s \\
& \leq \frac{1}{\underline{c}_{p}}\left(V_{p} \kappa+W_{p} \kappa+\bar{u}_{p}^{R}\right)=\frac{\Theta_{p} \kappa+\bar{u}_{p}^{R}}{\underline{c}_{p}}, \quad p \in \Lambda .
\end{aligned}
$$

In a similar way, we can obtain

$$
\sup _{t \in \mathbb{R}}\left|(T \varphi)_{p}(t)\right| \leq \frac{\Theta_{p} \kappa+\bar{u}_{p}^{l}}{\underline{c}_{p}}, \quad p \in \Lambda, l=I, J, K .
$$

It follows from (12), (13), and $\left(H_{4}\right)$ that 
which implies that $T \varphi \in \mathbb{X}^{*}$. Therefore, the mapping $T$ is a self-mapping from $\mathbb{X}^{*}$ to $\mathbb{X}^{*}$. Next, we show that $T: \mathbb{X}^{*} \rightarrow \mathbb{X}^{*}$ is a contraction mapping. In fact, for any $\varphi, \psi \in \mathbb{X}^{*}$, we have

$$
\begin{aligned}
& \sup _{t \in \mathbb{R}}\left|(T \varphi)_{p}^{R}(t)-(T \psi)_{p}^{R}(t)\right| \\
& \leq \sup _{t \in \mathbb{R}} \int_{-\infty}^{t} e^{-\int_{s}^{t} c_{p}(u) \mathrm{d} u}\left(\sum _ { q = 1 } ^ { n } ( \overline { a } _ { p q } ^ { R } + \overline { a } _ { p q } ^ { I } + \overline { a } _ { p q } ^ { I } + \overline { a } _ { p q } ^ { K } ) \left(\alpha_{q}^{R} \mid \varphi_{q}^{R}\left(s-\tau_{p q}(s)\right)\right.\right. \\
& -\psi_{q}^{R}\left(s-\tau_{p q}(s)\right)\left|+\alpha_{q}^{I}\right| \varphi_{q}^{I}\left(s-\tau_{p q}(s)\right)-\psi_{q}^{I}\left(s-\tau_{p q}(s)\right) \mid \\
& +\alpha_{q}^{J}\left|\varphi_{q}^{J}\left(s-\tau_{p q}(s)\right)-\psi_{q}^{J}\left(s-\tau_{p q}(s)\right)\right|+\alpha_{q}^{K} \mid \varphi_{q}^{K}\left(s-\tau_{p q}(s)\right) \\
& \left.-\psi_{q}^{K}\left(s-\tau_{p q}(s)\right) \mid\right)+\sum_{q=1}^{n}\left(\bar{b}_{p q}^{R}+\bar{b}_{p q}^{I}+\bar{b}_{p q}^{J}+\bar{b}_{p q}^{K}\right) \int_{0}^{\infty}\left|K_{p q}(u)\right| \\
& \times\left(\beta_{q}^{R}\left|\varphi_{q}^{R}(s-u)-\psi_{q}^{R}(s-u)\right|+\beta_{q}^{I}\left|\varphi_{q}^{I}(s-u)-\psi_{q}^{I}(s-u)\right|\right. \\
& \left.\left.+\beta_{q}^{J}\left|\varphi_{q}^{J}(s-u)-\psi_{q}^{J}(s-u)\right|+\beta_{q}^{K}\left|\varphi_{q}^{K}(s-u)-\psi_{q}^{K}(s-u)\right|\right) \mathrm{d} u\right) \mathrm{d} s \\
& \leq \sup _{t \in \mathbb{R}} \int_{-\infty}^{t} e^{-\int_{s}^{t} c_{p}(u) \mathrm{d} u}\left(\sum _ { q = 1 } ^ { n } ( \overline { a } _ { p q } ^ { R } + \overline { a } _ { p q } ^ { I } + \overline { a } _ { p q } ^ { I } + \overline { a } _ { p q } ^ { K } ) \left(\alpha_{q}^{R}+\alpha_{q}^{I}\right.\right. \\
& \left.+\alpha_{q}^{J}+\alpha_{q}^{K}\right)\|\varphi-\psi\|_{\mathbb{X}}+\sum_{q=1}^{n} \bar{K}_{p q}\left(\bar{b}_{p q}^{R}+\bar{b}_{p q}^{I}+\bar{b}_{p q}^{J}+\bar{b}_{p q}^{K}\right)\left(\beta_{q}^{R}+\beta_{q}^{I}\right. \\
& \left.\left.+\beta_{q}^{J}+\beta_{q}^{K}\right)\|\varphi-\psi\|_{\mathbb{X}}\right) \mathrm{d} s \\
& \leq \frac{1}{\underline{c}_{p}}\left(V_{p}+W_{p}\right)\|\varphi-\psi\|_{\mathbb{X}}=\frac{\Theta_{p}}{\underline{c}_{p}}\|\varphi-\psi\|_{\mathbb{X}} .
\end{aligned}
$$

In a similar way, we can obtain

$$
\sup _{t \in \mathbb{R}}\left|(T \varphi)_{p}^{l}(t)-(T \psi)_{p}^{l}(t)\right| \leq \frac{\Theta_{p}}{\underline{c}_{p}}\|\varphi-\psi\|_{\mathbb{X}}, \quad p \in \Lambda, l=I, J, K .
$$

It follows from (14), (15), and $\left(H_{4}\right)$ that

$$
\|T(\varphi)-T(\psi)\|_{\mathbb{X}} \leq \rho\|\varphi-\psi\|_{\mathbb{X}}
$$

Hence, $T$ is a contraction mapping from $\mathbb{X}^{*}$ to $\mathbb{X}^{*}$. Therefore, $T$ has a unique fixed point in $\mathbb{X}^{*}$, that is, (8) has a unique pseudo almost periodic solution in $\mathbb{X}^{*}$. The proof is complete.

By Remark 1, Theorem 1, we have the following.

Theorem 2 Suppose that $\left(S_{1}\right)-\left(S_{5}\right)$ hold, then system (1) has a unique pseudo almost periodic solution in $\mathbb{X}^{*}=\left\{\varphi \mid \varphi \in \mathbb{X},\|\varphi\|_{\mathbb{X}} \leq \kappa\right\}$. 
Definition 5 Let $x=\left(x_{1}^{R}, x_{1}^{I}, x_{1}^{J}, x_{1}^{K}, \ldots, x_{n}^{R}, x_{n}^{I}, x_{n}^{J}, x_{n}^{K}\right)^{T}$ be a solution of (8) with the initial value $\varphi=\left(\varphi_{1}^{R}, \varphi_{1}^{I}, \varphi_{1}^{J}, \varphi_{1}^{K}, \ldots, \varphi_{n}^{R}, \varphi_{n}^{I}, \varphi_{n}^{J}, \varphi_{n}^{K}\right)^{T} \in C\left((-\infty, 0], \mathbb{R}^{4 n}\right)$ and $y=\left(y_{1}^{R}, y_{1}^{I}, y_{1}^{J}, y_{1}^{K}, \ldots, y_{n}^{R}\right.$, $\left.y_{n}^{I}, y_{n}^{I}, y_{n}^{K}\right)^{T}$ be an arbitrary solution of system (8) with the initial value $\psi=\left(\psi_{1}^{R}, \psi_{1}^{I}, \psi_{1}^{J}, \psi_{1}^{K}\right.$, $\left.\ldots, \psi_{n}^{R}, \psi_{n}^{I}, \psi_{n}^{J}, \psi_{n}^{K}\right)^{T} \in C\left((-\infty, 0], \mathbb{R}^{4 n}\right)$. If there exist constants $\lambda>0$ and $M>0$ such that

$$
\|x(t)-y(t)\| \leq M\|\varphi-\psi\| e^{-\lambda t}, \quad t>0
$$

where

$$
\begin{aligned}
& \|x(t)-y(t)\|=\max _{p \in \Lambda}\left\{\left|x_{p}^{l}(t)-y_{p}^{l}(t)\right|, l \in E\right\}, \\
& \|\varphi-\psi\|_{0}=\max _{p \in \Lambda, l \in E}\left\{\sup _{s \in(-\infty, 0]}\left|\varphi_{p}^{l}(s)-\psi_{p}^{l}(s)\right|\right\} .
\end{aligned}
$$

Then the solution $x$ of system (8) is said to be globally exponentially stable.

Theorem 3 Under the assumptions of Theorem 1, system (8) has a unique pseudo almost periodic solution that is globally exponentially stable.

Proof From Theorem 1, we see that system (8) has a pseudo almost periodic solution $X^{*}=\left(X_{1}^{*}, X_{2}^{*}, \ldots, X_{n}^{*}\right)^{T}$ with initial value $\Phi^{*}=\left(\phi_{1}^{*}, \phi_{2}^{*}, \ldots, \phi_{n}^{*}\right)^{T}$. Suppose that $X=$ $\left(X_{1}, X_{2}, \ldots, X_{n}\right)^{T}$ is an arbitrary solution of system (8) with initial value $\Phi=\left(\phi_{1}, \phi_{2}, \ldots, \phi_{n}\right)^{T}$ and let $Z=X-X^{*}$, then we have

$$
\begin{aligned}
Z_{p}^{\prime}(t)= & -c_{p}(t) Z_{p}(t)+\sum_{q=1}^{n} A_{p q}(t)\left(F_{q}\left[t, z+x^{*}\right]-F_{q}\left[t, x^{*}\right]\right) \\
& +\sum_{q=1}^{n} B_{p q}(t) \int_{0}^{\infty} K_{p q}(u)\left(G_{q}\left[t, u, z+x^{*}\right]\right. \\
& \left.-G_{q}\left[t, u, x^{*}\right]\right) \mathrm{d} u, \quad p \in \Lambda .
\end{aligned}
$$

The initial condition of (16) is

$$
Z_{p}(s)=\psi_{p}(s)=\Phi_{p}(s)-\Phi_{p}^{*}(s), \quad s \in(-\infty, 0], p \in \Lambda
$$

For $p \in \Lambda$, we define $\Gamma_{p}$ as follows:

$$
\Gamma_{p}(\theta)=\theta-\underline{c}_{p}+V_{p} e^{\theta \bar{\tau}_{p q}}+W_{p}, \quad p \in \Lambda .
$$

From $\left(S_{5}\right)$, we have

$$
\Gamma_{p}(0)=-\underline{c}_{p}+V_{p}+W_{p}=-\underline{c}_{p}+\Theta_{p}<0
$$

and $\Gamma_{p}(\theta)$ is continuous on $[0,+\infty)$ and $\Gamma_{p}(\theta) \rightarrow+\infty$, as $\theta \rightarrow+\infty$. Hence, there exists $\xi_{p}>0$ such that $\Gamma_{p}\left(\xi_{p}\right)=0$ and $\Gamma_{p}(\theta)<0$ for $\theta \in\left(0, \xi_{p}\right), p \in \Lambda$. So, we can choose a positive constant $0<\lambda<\min \left\{\min _{p \in \Lambda} \xi_{p}, \min _{p \in \Lambda}\left\{\underline{c}_{p}\right\}\right\}$ such that

$$
\Gamma_{p}(\lambda)<0, \quad p \in \Lambda \text {. }
$$


Let $\gamma_{p}=V_{p} e^{\lambda \bar{\tau}_{p q}}+W_{p}, p \in \Lambda$. Then $\gamma_{p}<\underline{c}_{p}-\lambda, p \in \Lambda$. Take a constant $M$ such that

$$
M>\frac{\underline{c}_{p}-\lambda}{\gamma_{p}}>1, \quad p \in \Lambda,
$$

which yields

$$
\frac{1}{M}-\frac{\gamma_{p}}{\underline{c}_{p}-\lambda}<0, \quad p \in \Lambda
$$

Hence, for any $\epsilon>0$, it is obvious that

$$
\|Z(0)\|<\left(\|\varphi\|_{0}+\epsilon\right)
$$

and

$$
\|Z(t)\|<\left(\|\varphi\|_{0}+\epsilon\right) e^{-\lambda t}<M\left(\|\varphi\|_{0}+\epsilon\right) e^{-\lambda t}, \quad \forall t \in(-\infty, 0] .
$$

We claim that

$$
\|Z(t)\|<M\left(\|\varphi\|_{0}+\epsilon\right) e^{-\lambda t}, \quad \forall t>0 .
$$

Otherwise, there must exist some $p \in \Lambda$ and $\eta>0$ such that

$$
\left\{\begin{array}{l}
\left|Z_{p}(\eta)\right|=\|Z(\eta)\|=M\left(\|\varphi\|_{0}+\epsilon\right) e^{-\lambda \eta} \\
\|Z(t)\|<M\left(\|\varphi\|_{0}+\epsilon\right) e^{-\lambda t}, \quad t<\eta
\end{array}\right.
$$

Multiplying both sides of (16) by $e^{\int_{0}^{t} c_{p}(u) \mathrm{d} u}$ and integrating over $[0, t]$, we get

$$
\begin{aligned}
Z_{p}(t)= & Z_{p}(0) e^{-\int_{0}^{t} c_{p}(u) \mathrm{d} u}+\int_{0}^{t} e^{-\int_{s}^{t} c_{p}(u) \mathrm{d} u}\left(\sum _ { q = 1 } ^ { n } A _ { p q } ( s ) \left(F_{q}\left[s, z+x^{*}\right]\right.\right. \\
& \left.-F_{q}\left[s, x^{*}\right]\right)+\sum_{q=1}^{n} B_{p q}(s) \int_{0}^{\infty} K_{p q}(u)\left(G_{q}\left[s, u, z+x^{*}\right]\right. \\
& \left.\left.-G_{q}\left[s, u, x^{*}\right]\right) \mathrm{d} u\right) \mathrm{d} s .
\end{aligned}
$$

From this and (20), we get

$$
\begin{aligned}
& \left|z_{p}^{R}(\eta)\right| \\
& =\mid z_{p}^{R}(0) e^{-\int_{0}^{\eta} c_{p}(u) \mathrm{d} u}+\int_{0}^{\eta} e^{-\int_{s}^{\eta} c_{p}(u) \mathrm{d} u}\left(\sum _ { q = 1 } ^ { n } \left(a_{p q}^{R}(s) \hat{f}_{q}^{R}\left[s, x-x^{*}\right]\right.\right. \\
& \left.\quad-a_{p q}^{I}(s) \hat{f}_{q}^{I}\left[s, x-x^{*}\right]-a_{p q}^{J}(s) \hat{f}_{q}^{J}\left[s, x-x^{*}\right]-a_{p q}^{K}(s) \hat{f}_{q}^{K}\left[s, x-x^{*}\right]\right) \\
& \quad+\sum_{q=1}^{n}\left(b_{p q}^{R}(s) \int_{0}^{\infty} K_{p q}(u) \hat{g}_{q}^{R}\left[s, u, x-x^{*}\right]-b_{p q}^{I}(s) \int_{0}^{\infty} K_{p q}(u)\right.
\end{aligned}
$$




$$
\begin{aligned}
& \times \hat{g}_{q}^{I}\left[s, u, x-x^{*}\right]-b_{p q}^{J}(s) \int_{0}^{\infty} K_{p q}(u) \hat{g}_{q}^{J}\left[s, u, x-x^{*}\right] \\
& \left.\left.-b_{p q}^{K}(s) \int_{0}^{\infty} K_{p q}(u) \hat{g}_{q}^{K}\left[s, u, x-x^{*}\right]\right) \mathrm{d} u\right) \mathrm{d} s \\
& \leq\left(\|\varphi\|_{0}+\epsilon\right) e^{-\int_{0}^{\eta} c_{p}(u) \mathrm{d} u}+\int_{0}^{\eta} e^{-\int_{s}^{\eta} c_{p}(u) \mathrm{d} u}\left(\sum _ { q = 1 } ^ { n } \left(\bar{a}_{p q}^{R}+\bar{a}_{p q}^{I}\right.\right. \\
& \left.+\bar{a}_{p q}^{J}+\bar{a}_{p q}^{K}\right)\left(\alpha_{q}^{R}+\alpha_{q}^{I}+\alpha_{q}^{J}+\alpha_{q}^{K}\right) e^{\lambda \bar{\tau}_{p q}}+\sum_{q=1}^{n}\left(\bar{b}_{p q}^{R}+\bar{b}_{p q}^{I}\right. \\
& \left.\left.+\bar{b}_{p q}^{J}+\bar{b}_{p q}^{K}\right)\left(\beta_{q}^{R}+\beta_{q}^{I}+\beta_{q}^{J}+\beta_{q}^{K}\right) \int_{0}^{\infty}\left|K_{p q}(u)\right| e^{-\lambda s} e^{\lambda u} \mathrm{~d} u\right) \mathrm{d} s \\
& \times M\left(\|\varphi\|_{0}+\epsilon\right) \\
& \leq\left(\|\varphi\|_{0}+\epsilon\right) e^{-\lambda \eta} e^{-\int_{0}^{\eta}\left(c_{p}(u)-\lambda\right) \mathrm{d} u}+\int_{0}^{\eta} e^{-\int_{s}^{\eta} c_{p}(u) \mathrm{d} u}\left(\sum _ { q = 1 } ^ { n } \left(\bar{a}_{p q}^{R}\right.\right. \\
& \left.+\bar{a}_{p q}^{I}+\bar{a}_{p q}^{J}+\bar{a}_{p q}^{K}\right)\left(\alpha_{q}^{R}+\alpha_{q}^{I}+\alpha_{q}^{J}+\alpha_{q}^{K}\right) e^{\lambda \bar{\tau}_{p q}}+\sum_{q=1}^{n} \bar{K}_{p q}\left(\bar{b}_{p q}^{R}\right. \\
& \left.\left.+\bar{b}_{p q}^{I}+\bar{b}_{p q}^{J}+\bar{b}_{p q}^{K}\right)\left(\beta_{q}^{R}+\beta_{q}^{I}+\beta_{q}^{J}+\beta_{q}^{K}\right)\right) \mathrm{d} s M\left(\|\varphi\|_{0}+\epsilon\right) e^{-\lambda \eta} \\
& \leq\left(\|\varphi\|_{0}+\epsilon\right) e^{-\lambda \eta} e^{-\int_{0}^{\eta}\left(c_{p}(u)-\lambda\right) \mathrm{d} u}+\frac{1-e^{\left(\lambda-\underline{c}_{p}\right) \eta}}{\underline{c}_{p}-\lambda}\left(V_{p} e^{\lambda \bar{\tau}_{p q}}\right. \\
& \left.+W_{p}\right) M\left(\|\varphi\|_{0}+\epsilon\right) e^{-\lambda \eta} \\
& \leq M\left(\|\varphi\|_{0}+\epsilon\right) e^{-\lambda \eta}\left[\left(\frac{1}{M}-\frac{\gamma_{p}}{\underline{c}_{p}-\lambda}\right) e^{\left(\lambda-\underline{c}_{p}\right) \eta}+\frac{\gamma_{p}}{\underline{c}_{p}-\lambda}\right] \\
& <M\left(\|\varphi\|_{0}+\epsilon\right) e^{-\lambda \eta}
\end{aligned}
$$

where $\hat{f}_{q}^{l}\left[s, x-x^{*}\right] \triangleq f_{q}^{l}[s, x]-f_{q}^{l}\left[s, x^{*}\right], \hat{g}_{q}^{l}\left[s, u, x-x^{*}\right] \triangleq g_{q}^{l}[s, u, x]-g_{q}^{l}\left[s, u, x^{*}\right]$.

Similarly, we can get

$$
\left|z_{p}^{l}(\eta)\right|<M\left(\|\varphi\|_{0}+\epsilon\right) e^{-\lambda \eta}, \quad l=I, J, K
$$

It follows from (21) and (22) that

$$
\left|Z_{p}(\eta)\right|<M\left(\|\varphi\|_{0}+\epsilon\right) e^{-\lambda \eta}
$$

which contradicts the first equation of (20). Hence, (19) holds. Letting $\epsilon \rightarrow 0^{+}$, from (19), we have

$$
\|Z(t)\| \leq M\|\varphi\|_{0} e^{-\lambda t}, \quad \forall t>0
$$

Therefore, the pseudo almost periodic solution of system (8) is globally exponentially stable. The proof is complete. 
By Remark 1, Theorem 3, we have

Theorem 4 Suppose that $\left(S_{1}\right)-\left(S_{5}\right)$ hold, then system (1) has a unique pseudo almost periodic solution that is globally exponentially stable.

\section{An example}

In this section, we give an example to illustrate the feasibility and effectiveness of our results obtained in Sect. 3.

Example 1 Consider the following quaternion-valued system:

$$
\begin{aligned}
x_{p}^{\prime}(t)= & -c_{p}(t) x_{p}(t)+\sum_{q=1}^{2} a_{p q}(t) f_{q}\left(x_{q}\left(t-\tau_{p q}(t)\right)\right) \\
& +\sum_{q=1}^{2} b_{p q}(t) \int_{0}^{\infty} K_{p q}(u) g_{q}\left(x_{q}(t-u)\right) d u+u_{p}(t),
\end{aligned}
$$

where $p=1,2, x_{p}=x_{p}^{R}+i x_{p}^{I}+j x_{p}^{J}+k x_{p}^{K} \in \mathbb{Q}$, and the coefficients are taken as follows:

$$
\begin{aligned}
& c_{1}(t)=4+\cos (\sqrt{2} t), \quad c_{2}(t)=5-\sin t, \quad K_{p q}(u)=|\sin u| e^{-4 u}, \\
& f_{q}\left(x_{q}\right)=\frac{1}{4} \sin ^{2}\left(x_{q}^{R}+x_{q}^{J}\right)+i\left|x_{q}^{I}+x_{q}^{K}\right|+j \frac{1}{2} \sin x_{q}^{J}+k \frac{1}{2}\left(\left|x_{q}^{K}+1\right|+\left|x_{q}^{K}\right|-1\right), \\
& g_{q}\left(x_{q}\right)=\tan x_{q}^{R}+i \frac{1}{8} \sin ^{2}\left(x_{q}^{R}+x_{q}^{I}\right)+j\left|x_{q}^{J}\right|+k \frac{1}{4} \sin \left(\sqrt{2} x_{q}^{K}\right), \\
& a_{11}(t)=a_{12}(t)=0.032 \cos t+i 0.03 \sin (\sqrt{2} t)+j 0.028 \sin t+k 0.045 \cos t, \\
& a_{21}(t)=a_{22}(t)=0.04 \sin (\sqrt{2} t)+i 0.05 \sin (\sqrt{3} t)+j 0.036 \cos (\sqrt{2} t)+k 0.06 \sin t, \\
& b_{11}(t)=b_{12}(t)=0.4 \sin t+i 0.2 \cos (\sqrt{2} t)+j 0.3 \sin (\sqrt{3} t)+k 0.25 \cos t, \\
& b_{21}(t)=b_{22}(t)=0.5 \cos (\sqrt{3} t)+i 0.45 \sin t+j 0.35 \cos t+k 0.6 \sin (\sqrt{2} t), \quad \tau_{22}(t)=\sin ^{2} t \\
& \tau_{11}(t)=|\sin (2 t)|, \quad \tau_{12}(t)=\cos ^{2} t, \quad \tau_{21}(t)=|\sin (\sqrt{2} t)|, \\
& u_{1}(t)=u_{2}(t)=\cos t+i \sin (2 t)+j \sin (\sqrt{2} t)+k \cos (\sqrt{2} t) .
\end{aligned}
$$

By a simple calculation, we have

$$
\begin{aligned}
& \underline{c}_{1}=3, \quad \underline{c}_{2}=4, \quad \bar{K}_{p q}=\frac{1}{4}, \quad p, q=1,2, \\
& \alpha_{q}^{R}=\alpha_{q}^{J}=\frac{1}{2}, \quad \alpha_{q}^{I}=\alpha_{q}^{K}=\beta_{q}^{R}=\beta_{q}^{J}=1, \quad \beta_{q}^{I}=\beta_{q}^{K}=\frac{1}{4}, \\
& \bar{a}_{11}^{R}=\bar{a}_{12}^{R}=0.032, \quad \bar{a}_{11}^{I}=\bar{a}_{12}^{I}=0.03, \quad \bar{a}_{11}^{I}=\bar{a}_{12}^{J}=0.028, \\
& \bar{a}_{11}^{K}=\bar{a}_{12}^{K}=0.045, \quad \bar{a}_{21}^{R}=\bar{a}_{22}^{R}=0.04, \quad \bar{a}_{21}^{I}=\bar{a}_{22}^{I}=0.05, \\
& \bar{a}_{21}^{J}=\bar{a}_{22}^{J}=0.036, \quad \bar{a}_{21}^{K}=\bar{a}_{22}^{K}=0.06, \quad \bar{b}_{11}^{R}=\bar{b}_{12}^{R}=0.4, \\
& \bar{b}_{11}^{I}=\bar{b}_{12}^{I}=0.2, \quad \bar{b}_{11}^{J}=\bar{b}_{12}^{J}=0.3, \quad \bar{b}_{11}^{K}=\bar{b}_{12}^{K}=0.25, \\
& \bar{b}_{21}^{R}=\bar{b}_{22}^{R}=0.5, \quad \bar{b}_{21}^{I}=\bar{b}_{22}^{I}=0.45, \quad \bar{b}_{21}^{J}=\bar{b}_{22}^{I}=0.35, \quad \bar{b}_{21}^{K}=\bar{b}_{22}^{K}=0.6,
\end{aligned}
$$



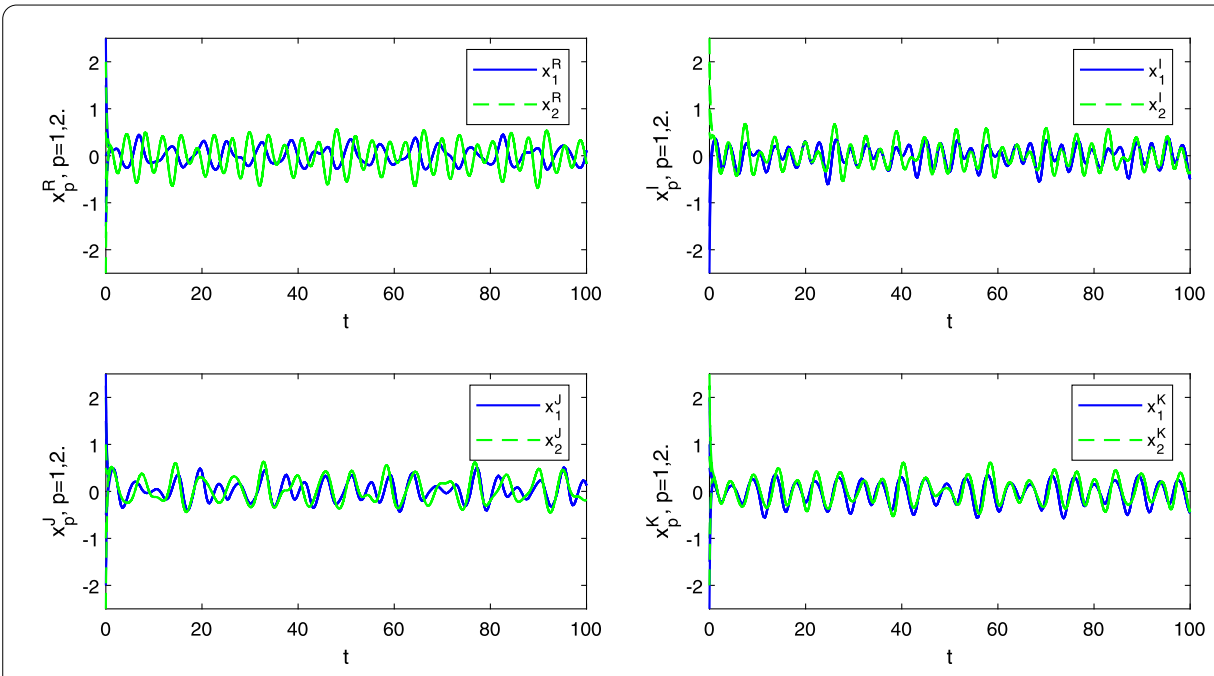

Figure 1 Transient states of four parts of QVNN (23) in Example 1

$$
\bar{u}_{1}^{R}=\bar{u}_{1}^{I}=\bar{u}_{1}^{J}=\bar{u}_{1}^{K}=\bar{u}_{2}^{R}=\bar{u}_{2}^{I}=\bar{u}_{2}^{I}=\bar{u}_{2}^{K}=1, \quad \bar{\tau}_{p q}=1, \quad p, q=1,2 .
$$

Take $\kappa=2$, then we have

$$
\begin{gathered}
\max \left\{\frac{2 \Theta_{1}+\bar{u}_{1}^{R}}{\underline{c}_{1}}, \frac{2 \Theta_{1}+\bar{u}_{1}^{I}}{\underline{c}_{1}}, \frac{2 \Theta_{1}+\bar{u}_{1}^{J}}{\underline{c}_{1}}, \frac{2 \Theta_{1}+\bar{u}_{1}^{K}}{\underline{c}_{1}}, \frac{2 \Theta_{2}+\bar{u}_{2}^{R}}{\underline{c}_{2}}, \frac{2 \Theta_{2}+\bar{u}_{2}^{I}}{\underline{c}_{2}},\right. \\
\left.\frac{2 \Theta_{2}+\bar{u}_{2}^{J}}{\underline{c}_{2}}, \frac{2 \Theta_{2}+\bar{u}_{2}^{K}}{\underline{c}_{2}}\right\} \approx\{1.8327,1.9955\}=1.9955 \leq \kappa=2
\end{gathered}
$$

and

$$
\max \left\{\frac{\Theta_{1}}{\underline{c}_{1}}, \quad \frac{\Theta_{2}}{\underline{c}_{2}}\right\} \approx\{0.7492,0.8728\}=0.8728=\rho<1 .
$$

It is easy to check that all the assumptions in Theorem 4 are satisfied. Therefore, we obtain that (23) has a pseudo almost periodic solution that is globally exponentially stable (see Fig. 1).

Remark 2 The results obtained in [13-15, 21, 27-34] cannot be applied to obtain that system (23) has a unique pseudo almost periodic solution that is globally exponentially stable.

\section{Conclusion}

In this paper, we have established the existence and global exponential stability of pseudo almost periodic solutions of QVCNNs with discrete and distributed delays. An example has been given to demonstrate the effectiveness of our results. This is the first time to study the pseudo almost periodic oscillation for QVCNNs with discrete and distributed delays. Furthermore, the method of this paper can be used to study other types of quaternionvalued neural networks. 


\section{Funding}

The first author was supported by the National Natural Science Foundation of China (No. 11861072 and No. 11361072) and the Science Research Fund of Education Department of Yunnan Province of China (No. 2017YJS111). The second author was supported by the National Natural Science Foundation of China (No. 11861072 and No. 11361072).

\section{Competing interests}

The authors declare that they have no competing interests.

\section{Authors' contributions}

The authors have made the same contribution. All authors read and approved the final manuscript.

\section{Publisher's Note}

Springer Nature remains neutral with regard to jurisdictional claims in published maps and institutional affiliations.

Received: 24 May 2018 Accepted: 6 September 2018 Published online: 19 September 2018

\section{References}

1. Chua, L.O., Yang, L.: Cellular neural networks: applications. IEEE Trans. Biomed. Circuits Syst. 35(10), 1273-1290 (1988)

2. Gilli, M., Biey, M., Checco, P.: Equilibrium analysis of cellular neural networks. IEEE Trans. Circuits Syst. I, Regul. Pap. 51(5), 903-912 (2004)

3. Guo, Z., Wang, J., Yan, Z:: Attractivity analysis of memristor-based cellular neural networks with time-varying delays. IEEE Trans. Neural Netw. Learn. Syst. 25(4), 704-717 (2014)

4. Liu, B.: Global exponential convergence of non-autonomous cellular neural networks with multi-proportional delays. Neurocomputing 191, 352-355 (2016)

5. Park, J.H.: Global exponential stability of cellular neural networks with variable delays. Appl. Math. Comput. 183(2), 1214-1219 (2006)

6. Lu, X., Chen, W.H., Du, R.: Stability analysis of delayed cellular neural networks with impulsive effects. IMA J. Math. Control Inf. 28(4), 487-506 (2011)

7. Long, S., Xu, D.: Global exponential stability of non-autonomous cellular neural networks with impulses and time-varying delays. Commun. Nonlinear Sci. Numer. Simul. 18(6), 1463-1472 (2013)

8. Zhang, J., Gui, Z.: Periodic solutions of nonautonomous cellular neural networks with impulses and delays. Nonlinear Anal., Real World Appl. 10(3), 1891-1903 (2009)

9. $\mathrm{Xu}, \mathrm{Y}$.: New results on almost periodic solutions for CNNs with time-varying leakage delays. Neural Comput. Appl. 25(6), 1293-1302 (2014)

10. Zhang, H.: Existence and stability of almost periodic solutions for CNNs with continuously distributed leakage delays. Neural Comput. Appl. 24(5), 1135-1146 (2014)

11. Zhou, Q.: Weighted pseudo anti-periodic solutions for cellular neural networks with mixed delays. Asian J. Contro 19(4), 1557-1563 (2017). https://doi.org/10.1002/asjc.1468

12. Li, Y:: Periodic solutions of non-autonomous cellular neural networks with impulses and delays on time scales. IMA J. Math. Control Inf. 31(2), 273-293 (2013)

13. Liu, B.: Pseudo almost periodic solutions for CNNs with continuously distributed leakage delays. Neurocomputing $148,445-454(2015)$

14. Yu, Y.: Exponential stability of pseudo almost periodic solutions for cellular neural networks with multi-proportional delays. Neural Process. Lett. 45(1), 141-151 (2017)

15. $\mathrm{Xu}, \mathrm{Y}$.: Exponential stability of pseudo almost periodic solutions for neutral type cellular neural networks with d operator. Neural Process. Lett. 46, 329-342 (2017)

16. Kobayashi, M.: Quaternionic Hopfield neural networks with twin-multistate activation function. Neurocomputing 267, 304-310 (2017)

17. Matsui, N., Isokawa, T., Kusamichi, H., Peper, F., Nishimura, H.: Quaternion neural network with geometrical operators, J. Intell. Fuzzy Syst. 15, 149-164 (2004)

18. Liu, Y., Zhang, D., Lu, J.: Global exponential stability for quaternion-valued recurrent neural networks with time-varying delays. Nonlinear Dyn. 87(1), 553-565 (2016)

19. Liu, Y., Zhang, D., Lou, J., Lu, J., Cao, J.: Stability analysis of quaternion-valued neural networks: decomposition and direct approaches. IEEE Trans. Neural Netw. Learn. Syst. 29(9), 4201-4211 (2017). https://doi.org/10.1109/TNNLS.2017.2755697

20. Chen, X., Li, Z., Song, Q., Hu, J., Tan, Y.: Robust stability analysis of quaternion-valued neural networks with time delays and parameter uncertainties. Neural Netw. 91, 55-65 (2017)

21. Li, Y., Meng, X.: Existence and global exponential stability of pseudo almost periodic solutions for neutral type quaternion-valued neural networks with delays in the leakage term on time scales. Complexity 2017, Article ID 9878369 (2017). https://doi.org/10.1155/2017/9878369

22. Liu, Y., Zhang, D., Lu, J., Cao, J.: Global $\mu$-stability criteria for quaternion-valued neural networks with unbounded time-varying delays. Inf. Sci. 360, 273-288 (2016)

23. Shu, H., Song, Q., Liu, Y., Zhao, Z., Alsaadi, F.E.: Global $\mu$-stability of quaternion-valued neural networks with non-differentiable time-varying delays. Neurocomputing 247, 202-212 (2017)

24. Li, Y., Qin, J.: Existence and global exponential stability of periodic solutions for quaternion-valued cellular neural networks with time-varying delays. Neurocomputing 292, 91-103 (2018)

25. Li, Y., Meng, X., Ye, Y.: Almost periodic synchronization for quaternion-valued neural networks with time-varying delays. Complexity 2018, Article ID 6504590 (2018). https://doi.org/10.1155/2018/6504590

26. Hakl, R., Pinto, M., Tkachenko, V., Trofimchuk, S.: Almost periodic evolution systems with impulse action at state-dependent moments. J. Math. Anal. Appl. 446(1), 1030-1045 (2017) 
27. Abbas, S.: Pseudo almost periodic sequence solutions of discrete time cellular neural networks. Nonlinear Anal., Model. Control 14(3), 283-301 (2009)

28. Pinto, M.: Pseudo-almost periodic solutions of neutral integral and differential equations with applications. Nonlinear Anal., Theory Methods Appl. 72(12), 4377-4383 (2010)

29. Arbi, A., Cao, J.: Pseudo-almost periodic solution on time-space scales for a novel class of competitive neutral-type neural networks with mixed time-varying delays and leakage delays. Neural Process. Lett. 46(2), 719-745 (2017)

30. Li, Y., Yang, L., Li, B.: Existence and stability of pseudo almost periodic solution for neutral type high-order Hopfield neural networks with delays in leakage terms on time scales. Neural Process. Lett. 44(3), 603-623 (2016)

31. Li, Y., Zhao, L.: Weighted pseudo-almost periodic functions on time scales with applications to cellular neural networks with discrete delays. Math. Methods Appl. Sci. 40(6), 1905-1921 (2017)

32. Liu, B., Tunç, C.: Pseudo almost periodic solutions for CNNs with leakage delays and complex deviating arguments. Neural Comput. Appl. 26(2), 429-435 (2015)

33. Li, Y., Meng, X., Xiong, L.: Pseudo almost periodic solutions for neutral type high-order Hopfield neural networks with mixed time-varying delays and leakage delays on time scales. Int. J. Mach. Learn. Cybern. 8(6), 1915-1927 (2017)

34. Tunç, C.: Pseudo almost periodic solutions for HCNNs with time-varying leakage delays. Moroccan J. Pure Appl. Anal. 1(1), 51-69 (2015)

35. Domoshnitsky, A., Fridman, E.: A positivity-based approach to delay-dependent stability of systems with large time-varying delays. Syst. Control Lett. 97, 139-148 (2016)

36. Domoshnitsky, A., Shklyar, R.: Positivity for non-Metzler systems and its applications to stability of time-varying delay systems. Syst. Control Lett. 118,44-51 (2018)

37. Fink, A.M.: Almost Periodic Differential Equations. Springer, Berlin (1974)

38. Zhang, C.: Pseudo almost periodic solutions of some differential equations. J. Math. Anal. Appl. 181(1), 62-76 (1994)

39. Zhang, C.: Pseudo almost periodic solutions of some differential equations, II. J. Math. Anal. Appl. 192(2), 543-561 (1995). https://doi.org/10.1006/jmaa.1995.1189

40. Zhang, C.: Almost Periodic Type Functions and Ergodicity. Science Press, Beijing (2003)

\section{Submit your manuscript to a SpringerOpen ${ }^{\circ}$ journal and benefit from:}

- Convenient online submission

- Rigorous peer review

Open access: articles freely available online

- High visibility within the field

- Retaining the copyright to your article

Submit your next manuscript at $\boldsymbol{~ s p r i n g e r o p e n . c o m ~}$ 